\title{
Eficácia da compressão pneumática intermitente (CPI) nos membros inferiores sobre o fluxo sanguíneo das veias femorais comuns
}

\author{
Efficacy of intermittent pneumatic compression (IPC) in lower \\ limbs on the blood flow of common femoral veins
}

Marcondes Figueiredo ${ }^{1}$, Patrícia Polizel Simão², Beethoven Marques Alves Pereira ${ }^{3}$, Nilson Penha-Silva ${ }^{4}$

\section{Resumo}

Contexto: A profilaxia do tromboembolismo venoso pode ser feita por métodos farmacológicos ou de forma mecânica, com o uso de meias antitrombo e compressão pneumática (CPI). A CPI é um método mecânico de profilaxia que merece melhor atenção da comunidade médica.

Objetivo: Avaliar o efeito do uso de compressão pneumática intermitente (CPI) nos pés, pernas e coxas de adultos saudáveis sobre o fluxo sanguíneo nas veias femorais comuns.

Métodos: A amostra foi constituída de 10 voluntários (sete mulheres e três homens) adultos (20-40 anos), sem antecedente de tromboembolismo venoso. Após repouso (10 min) em decúbito dorsal, o fluxo sanguíneo era medido (três vezes) por ultra-sonografia vascular na veia femoral, a $1 \mathrm{~cm}$ acima da junção safeno-femoral, em ambos os membros, sem controle e com CPI no pé, perna e na coxa. A compressão (130 mmHg no pé e $45 \mathrm{mmHg}$ na perna e na coxa) foi aplicada em ciclos de 11 segundos de compressão e 20-60 segundos de esvaziamento. As aferições de fluxo foram realizadas no pico de fluxo do ciclo de compressão. Os valores foram comparados por análise de variância (teste de Tukey), com $\mathrm{p}<0,05$ indicando diferença estatisticamente significante.

Resultado: A utilização de CPI nos membros inferiores, esquerdo e direito, promoveu elevações percentuais relativas no fluxo venoso femoral de 37,6 e $70,8 \%$ (pés), 143,9 e 164,7\% (pernas) e 132,6 e 128,9\% (coxas), respectivamente. As variações foram estatisticamente significantes para as aplicações nas pernas e coxas.

Conclusão: A CPI melhora o fluxo sanguíneo quando aplicada na perna ou na coxa.

Palavras-chave: Dispositivo de compressão pneumática intermitente, trombose venosa, ultra-sonografia, veia femoral.

\begin{abstract}
Background: Prophylaxis of venous thromboembolism, a very common post-surgical complication, can be made pharmacologically or via mechanical methods, such as the use of anti-thrombosis socks and intermittent pneumatic compression (IPC). IPC is a mechanical method of prophylaxis that deserves the attention of the medical community.

Objective: To evaluate the effect of IPC on the blood flow of common femoral veins in feet, legs and thighs of healthy adults.

Methods: The sample was comprised of 10 volunteers (seven females and three males), adults (20-40 years), without history of venous thromboembolism. After a rest period $(10 \mathrm{~min})$, with the patient in the supine position, blood flow was measured (three times) via vascular ultrasonography at the femoral vein, $1 \mathrm{~cm}$ above of the saphenofemoral junction, in both limbs, without (control) and with IPC in the patient's foot, leg and thigh. Compression $(130 \mathrm{mmHg}$ on foot and $45 \mathrm{mmHg}$ on leg and thigh) was applied at compression cycles of 11 seconds and emptying cycles of 20-60 seconds. Blood flow evaluations were performed at compression cycle peaks. Values were compared by analysis of variance (Tukey test), $\mathrm{p}<0.05$ indicating statistically significant difference.
\end{abstract}

Results: Use of IPC on the left and right lower limbs caused a percentage increase in the femoral blood flow of 37.6 and $70.8 \%$ (feet), 143.9 and $164.7 \%$ (legs), and 132.6 and $128.9 \%$ (thighs), respectively. Variations were statistically significant for application in legs and thighs.

Conclusion: Use of IPC improves blood flow when applied in legs or thighs.

Keywords: Intermittent pneumatic compression, venous thrombosis, ultrasonography, femoral vein.

1. Doutor, Angiologia e Cirurgia Vascular, Universidade Federal de São Paulo (UNIFESP), São Paulo, SP.

2. Médica, ultra-sonografista vascular.

3. Graduando, Faculdade de Medicina, Universidade Federal de Uberlândia (UFU), Uberlândia, MG.

4. Pós-doutor em Bioquímica, Southern Illinois University, Carbondale, IL, EUA. University of Texas Medical Branch (UTMB), Galveston, Texas, EUA. Professor titular, Instituto de Genética e Bioquímica, UFU, Uberlândia, MG.

Não foram declarados conflitos de interesse associados à publicação deste artigo.

Artigo submetido em 15.02.08, aceito em 03.09.08.

J Vasc Bras. 2008;7(4):321-324.

Copyright( 2008 by Sociedade Brasileira de Angiologia e de Cirurgia Vascular 


\section{Introdução}

A embolia pulmonar (EP) é a principal causa de morte em pacientes internados. A maioria das embolias fatais é oriunda de trombose venosa profunda (TVP) nos membros inferiores ${ }^{1}$.

A profilaxia do tromboembolismo venoso pode ser feita por métodos farmacológicos, principalmente com uso de heparina de baixo peso molecular ${ }^{2-4}$, ou por métodos mecânicos, como o uso da meia antitrombo ${ }^{3}$ e da compressão pneumática intermitente $(\mathrm{CPI})^{3,5-8}$.

A CPI foi inicialmente utilizada em pacientes para prevenir a trombose venosa após tratamento cirúrgi$\mathrm{co}^{8,9}$, mas passou também a ser usada durante o tratamento operatório e em períodos de imobilidade do paciente $^{10}$. Sua ação é fundamentada no aumento do fluxo venoso das veias do membro inferior e na indução da atividade fibrinolítica na corrente sanguínea ${ }^{10}$.

O objetivo deste trabalho foi avaliar, por ultrasonografia vascular, o efeito do uso de CPI nos pés, pernas e coxas sobre o fluxo venoso na veia femoral de adultos jovens saudáveis.

\section{Casuística}

Este trabalho está de acordo com a resolução 196/96 do Conselho Nacional de Saúde e a Declaração de Helsinki, tendo sido previamente aprovado pelo Comitê de Ética do pelo Comitê de Ética do Hospital Santa Catarina, em Uberlândia, Minas Gerais.

A amostra foi constituída de 10 voluntários (sete mulheres e três homens) adultos (20-40 anos), sem antecedentes de tromboembolismo venoso. O fluxo sanguíneo femoral foi aferido por ultra-sonografia vascular (aparelho ATL ${ }^{\circledR}$ HDI 5000 e transdutor linear de 4-7 $\mathrm{MHz}$ ) na veia femoral comum, a aproximadamente 1 $\mathrm{cm}$ acima da junção safeno-femoral, sempre pelo mesmo examinador. No total foram analisados 20 membros. As aferições foram feitas após repouso de 10 min em decúbito dorsal, inicialmente sem compressão (controle), e depois com compressão de $130 \mathrm{mmHg}$ no pé e $45 \mathrm{mmHg}$ na perna e coxa (com auxílio do aparelho SCD EXPRESS $^{\circledR}$ ), com ciclos de 11 segundos de compressão e de 20 a 60 segundos de esvaziamento. Esses aparelhos são seqüenciais, ou seja, as câmaras são infladas ordenhando o fluxo sanguíneo no sentido do coração.
Os valores do fluxo sanguíneo femoral foram inicialmente submetidos a teste de avaliação da normalidade (Kolmogorov-Smirnov). Os valores entre os diferentes grupos foram depois comparados por análise de variância (teste de Tukey), com $\mathrm{p}<0,05$ indicando diferença estatisticamente significante.

\section{Resultados}

Em todas as situações consideradas, os valores de fluxo femoral seguiram distribuições gaussianas $(\alpha=$ $0,05)$, segundo o teste de Kolmogorov-Smirnov. A Tabela 1 apresenta os valores obtidos de fluxo femoral. Em todas as situações, os valores obtidos para perna e coxa foram significantemente maiores que aquele obtido para o controle.

Como não houve diferenças estatisticamente significantes entre os membros direito e esquerdo, os dados foram agrupados e outra análise foi feita. Essa análise mostrou que os valores de fluxo foram maiores quando a CPI foi aplicada na perna e coxa em relação ao pé e ao controle, embora não tenha havido diferença significante entre os valores obtidos para perna e coxa (Tabela 1).

\section{Discussão}

O método utilizado neste trabalho foi caso-controle, ou seja, o paciente foi o controle dele mesmo antes de receber aplicação da CPI. A aplicação de CPI na perna e coxa direitas de pacientes jovens e saudáveis (18 mulheres e oito homens) foi avaliada por Flam e colegas ${ }^{10}$. Esse estudo mostrou um aumento maior de fluxo quando a CPI era aplicada na perna em relação à coxa.

A compressão pneumática pode ser seqüencial ou não-seqüencial ${ }^{11,12}$.

Este trabalho comparou o ponto máximo de fluxo no ciclo de compressão entre três diferentes regiões (pé, perna e coxa) dos membros inferiores direito e esquerdo, utilizando compressores do tipo seqüencial, um para o pé e outro para perna e coxa, com mudança apenas da perneira entre um local e outro.

A compressão pneumática intermitente tem como principal mecanismo de ação a eliminação da estase venosa, que é a diminuição tanto da velocidade como do volume de fluxo sanguíneo no interior da veia. A 
Tabela 1 - Fluxo venoso femoral (mL/min) sem (controle) e com CPI no pé, perna e coxa, em média \pm desvio padrão e em variação percentual relativa ao controle

\begin{tabular}{|c|c|c|c|c|}
\hline CPI & $\begin{array}{c}\text { Membro inferior } \\
\text { esquerdo }\end{array}$ & Membro inferior direito & $\begin{array}{c}\text { Membros inferiores } \\
\text { (ambos) }\end{array}$ & p (entre membros) \\
\hline Controle (sem CPI) & $\begin{array}{c}256,49 \pm 126,23^{\mathrm{cd}} \\
(\mathrm{n}=10)\end{array}$ & $\begin{array}{c}253,95 \pm 98,74^{\mathrm{cd}} \\
(\mathrm{n}=10)\end{array}$ & $\begin{array}{c}255,22 \pm 110,31^{\mathrm{cd}} \\
(\mathrm{n}=20)\end{array}$ & ns \\
\hline Pé & $\begin{array}{c}353,04 \pm 123,47^{\mathrm{cd}} \\
(\mathrm{n}=10) \\
37,6 \%\end{array}$ & $\begin{array}{c}433,74 \pm 271,38 \\
(\mathrm{n}=10) \\
70,8 \%\end{array}$ & $\begin{array}{c}393,39 \pm 209,33^{\mathrm{cd}} \\
(\mathrm{n}=20) \\
54,2 \%\end{array}$ & ns \\
\hline Perna & $\begin{array}{c}625,55 \pm 205,97^{\mathrm{abd}} \\
(\mathrm{n}=10) \\
143,9 \%\end{array}$ & $\begin{array}{c}672,16 \pm 328,23^{\mathrm{a}} \\
(\mathrm{n}=10) \\
164,7 \%\end{array}$ & $\begin{array}{c}648,86 \pm 267,77^{\mathrm{ab}} \\
(\mathrm{n}=20) \\
154,3 \%\end{array}$ & ns \\
\hline Coxa & $\begin{array}{c}596,53 \pm 267,51^{\mathrm{abc}} \\
(\mathrm{n}=10) \\
132,6 \%\end{array}$ & $\begin{array}{c}581,25 \pm 235,50^{\mathrm{a}} \\
(\mathrm{n}=10) \\
128,9^{\circ} \%\end{array}$ & $\begin{array}{c}588,89 \pm 245,42^{\mathrm{ab}} \\
(\mathrm{n}=20) \\
130,7 \%\end{array}$ & ns \\
\hline
\end{tabular}

ns = não-significante.

$\mathrm{p}<0,05$ indicando diferença estatisticamente significante (teste de Tukey) em relação ao controle ${ }^{\mathrm{a}}$, pé $^{\mathrm{b}}$, perna $^{\mathrm{c}}$ e $\operatorname{coxa}^{\mathrm{d}}$.

estase venosa é considerada o principal fator desencadeante da trombose venosa profunda ${ }^{13}$. O tamanho, a duração e o perfil do aumento do fluxo venoso dependerão do sistema de compressão utilizado ${ }^{14}$. Quando a compressão é aplicada no membro, a zona comprimida acelera o fluxo do sangue, facilitando o esvaziamento e prevenindo a estase ${ }^{15}$.

De fato, o uso de CPI no pós-operatório reduz o tromboembolismo venoso em $60 \%$. Ela aumenta o fluxo venoso da veia femoral quando aplicada no pé, perna e $\operatorname{coxa}^{16}$, mas é mais eficaz quando aplicada na perna ou coxa em relação ao pé ${ }^{16,17}$.

No presente estudo, nós também avaliamos o uso de CPI seqüencial no pé, perna e coxa. As aplicações de CPI na perna e na coxa, mas não no pé, aumentaram significantemente o fluxo da veia femoral (Tabela 1). Embora o aumento do fluxo decorrente da aplicação do pé não tenha sido significante, as aplicações de CPI na perna e coxa produziram elevações significantes no fluxo femoral em relação à aplicação no pé, o que é concordante com descrições prévias da literatura ${ }^{16,17}$. Apesar da CPI estar estabelecida como um método mecânico de profilaxia do tromboembolismo venoso ${ }^{18,19}$, a adesão da comunidade médica a esse procedimento é baixa $^{20-22}$.
É, portanto, necessário conscientizar a classe médica da importância da CPI. Uma mudança de postura da comunidade médica poderia diminuir a mortalidade por embolia pulmonar, que chega a ser de 50.000 casos por ano nos EUA, onde surgem a cada ano 160 novos casos de trombose venosa profunda para cada 100.000 habitantes ${ }^{18}$.

\section{Conclusão}

A aplicação de CPI na perna ou coxa aumenta o fluxo sanguíneo, diminuindo a estase.

\section{Referências}

1. Howard A, Howard DPJ, Davies AH. Introduction to venous thromboembolism and prophylaxis. Phlebology. 2006;21:1-10.

2. Nicolaides AN, Dupont PA, Desai S, Lewis JD, Douglas JN. Small doses of subcutaneous heparin in preventing postoperative deep venous thrombosis. Am Heart J. 1974;87:261-3.

3. Kalodiki EP, Hoppensteadt DA, Nicolaides AN, et al. Deep venous thrombosis prophylaxis with low molecular weight heparin and elastic compression in patients having total hip replacement. A randomised controlled trial. Int Angiol. 1996;15:162-8.

4. Howard A, Zaccagnini D, Williams A, et al. A protocol of low molecular weight heparin and antiembolic stockings abolishes deep vein thrombosis in low and moderate-risk patients and reduces it in high-risk surgical patients. Br J Surg. 90:73-73. 
5. Nicolaides AN, Fernandes e Fernandes J, Pollock AV. Intermittent sequential pneumatic compression of the legs in the prevention of venous stasis and postoperative deep venous thrombosis. Surgery. 1980;87:69-76.

6. Nicolaides AN, Miles C, Hoare M, Jury P, Helmis E, Venniker $\mathrm{R}$. Intermittent sequential pneumatic compression of the legs and thromboembolism-deterrent stockings in the prevention of postoperative deep venous thrombosis. Surgery. 1983;94:21-5.

7. Kakkos SK, Daskalopoulou SS, Daskalopoulos ME, Nicolaides AN, Geroulakos G. Review on the value of graduated elastic compression stockings after deep vein thrombosis. Thromb Haemost. 2006;96:441-5.

8. Urbankova J, Quiroz R, Kucher N, Goldhaber SZ. Intermittent pneumatic compression and deep vein thrombosis prevention in postoperative patients. Thromb Haemost. 2005;94:1181-5.

9. Hills NH, Pflug JJ, Jeyasingh K, Boardman L, Calnan JS. Prevention of deep vein thrombosis by intermittent pneumatic compression of calf. Br Med J. 1972;1:131-5.

10. Flam E, Berry S, Coyle A, Dardik H, Raab L. Blood-flow augmentation of intermittent pneumatic compression systems used for prevention of deep vein thrombosis prior to surgery. Am J Surg. 1996;171:312-5.

11. Kakkos SK, Griffin M, Geroulakos G, Nicolaides AN. The efficacy of new portable sequential compression device (SCD express) in preventing venous stasis. J Vasc Surg. 2005;42:296-303.

12. Kakkos SK, Nicolaides AN, Griffin M, Geroulakos G. Comparison of two intermittent pneumatic compression systems: a hemodynamic study. Int Angiol. 2005;24:330-5.

13. Maffei FH, Rollo HA.Trombose venosa profunda dos membros inferiores. In: Maffei FH, Lastória S, Yoshida WB, Rollo HA, editores. Doenças vasculares periféricas. $3^{\mathrm{a}}$ ed. Rio de Janeiro: Medsi; 2001. p. 1363-86.

14. Morris RJ, Woodcoock JP. Evidence-based compression: prevention of stasis and deep vein thrombosis. Ann Surg. 2004;239:162-71.

15. Chen AH, Frangos SG, Kilaru S, Sumpio BE. Intermittent pneumatic compression devices - physiological mechanisms of actions. Eur J Endovasc Surg. 2001;21:383-92.
16. Delis KT, Slimani G, Hafez HM, Nicolaides AN. Enhancing venous out flow in the lower limb with intermittent pneumatic compression. A comparative haemodynamic analysis on the effect of foot vs. calf vs. foot and calf compression. Eur J Vasc Endovasc Surg. 2000;19:250-60.

17. Keith SL, McLaughlin DJ, Anderson FA Jr, et al. Do graduated compression stockings and pneumatic boots have an additive effect on the peak velocity of venous blood flow? Arch Surg. 1992;127:727-30.

18. Cardiovascular Disease Educational and Research Trust; Cyprus Cardiovascular Disease Educational and Research Trust; European Venous Forum; International Surgical Thrombosis Forum; International Union of Angiology; Union Internationale de Phlébologie. Prevention and treatment of venous thromboembolism. International Consensus Statement (guidelines according to scientific evidence). Int Angiol. 2006;25:101-61.

19. Nutescu EA. Assessing, preventing, and treating venous thromboembolism: evidence-based approaches. Am J Health Syst Pharm. 2007;64:S5-13.

20. Engelhorn ALV, Garcia ACF, Cassou AF, Birckholz L, Engelhorn CA. Profilaxia da trombose venosa profunda: estudo epidemiológico em um hospital escola. J Vasc Bras. 2002;1:97-102.

21. Garcia ACS, Souza BV, Volpato DE, et al. Realidade do uso da profilaxia para trombose venosa profunda: da teoria à prática. J Vasc Bras. 2005;4:35-41.

22. Stratton MA, Anderson FA, Bussey HI, et al. Prevention of venous thromboembolism: adherence to the 1995 American College of Chest Physicians consensus guidelines for surgical patients. Arch Intern Med. 2000;160:334-40.

Correspondência:

Marcondes Figueiredo

Rua Marques Povoa, 88

CEP 38400-438 - Uberlândia, MG

E-mail: drmarcondes@gmail.com 